\title{
Carnets
}

Revue électronique d'études françaises de l'APEF

Deuxième série - 19 | 2020

Petite fabrique d'interprètes

\section{La géographie des identités}

Dany Laferrière, Pays sans chapeau

\section{Anikó Ádám}

\section{CpenEdition}

Journals

Édition électronique

URL : http://journals.openedition.org/carnets/11646

DOI : $10.4000 /$ carnets. 11646

ISSN : 1646-7698

Éditeur

APEF

Référence électronique

Anikó Ádám, "La géographie des identités », Carnets [En ligne], Deuxième série - 19 | 2020, mis en ligne le 31 mai 2020, consulté le 23 décembre 2020. URL : http://journals.openedition.org/carnets/ 11646 ; DOI : https://doi.org/10.4000/carnets. 11646

Ce document a été généré automatiquement le 23 décembre 2020.

\section{(c) (7) \&}

Carnets est mis à disposition selon les termes de la licence Creative Commons - Atribution - Pas d'utilisation commerciale 4.0 International. 


\title{
La géographie des identités
}

\author{
Dany Laferrière, Pays sans chapeau
}

\author{
Anikó Ádám
}

1 Dany Laferrière, écrivain québécois, est un des plus significatifs représentants des prises d'identités différentes: psychologique, nationale et professionnelle. Il paraît nécessaire de préciser néanmoins que la différence entre ces identités apparaît momentanément sur un axe horizontal (d'après le suivi du parcours autobiographique) et vertical (selon la sensibilité psychologique de l'écrivain).

2 Né à Port au Prince le 17 avril 1953, notre auteur a grandi au village de Petit-Goâve. Comme il est journaliste, sa vie est menacée par la dictature. Il s'exile donc en 1976 au Canada. C'est à Montréal que l'auteur trouve une terre d'asile et occupe plusieurs emplois, puis il débute au Québec tout en se définissant une naissance physique et une naissance d'écrivain: «Je suis né physiquement en Haïti, mais je suis né comme écrivain à Montréal. »

3 Après avoir publié son premier livre Comment faire l'amour avec un nègre sans se fatiguer (1985), il devient très vite connu comme écrivain québécois et il se consacre à la littérature. L'identité naturelle de Dany Laferrière se complète alors de l'identité professionnelle au Québec, dans le pays dont la survivance culturelle est notamment confrontée au multiculturalisme. Depuis le début des années 1990, Dany Laferrière vit à Miami une dizaine de mois par année.

4 Arrivant au Canada, notre écrivain se trouve entre deux espaces culturels, et se forge, en écrivant et en lisant, une identité interculturelle. Tout en reconnaissant son américanité nègre, à travers la rupture avec son passé, il se charge d'une identité interculturelle qui tourne très vite en identité pluriculturelle par le métissage syncrétique des fragments de la nouvelle culture américaine et de l'ancienne culture européenne. C'est seulement avec le retour en Haïti qu'il récupère son identité personnelle et universelle et qu'il arrive aux confins de toute culture absorbée pour rester en transe. Il transforme alors en écrivain transculturel et transaméricain.

5 La présente étude vise à offrir l'analyse des identités à partir de son roman intitulé Pays sans chapeau (Editions Lanctôt, 1996) où l'auteur tente de regagner et de ressaisir son pays natal, et son identité volée par la dictature. Il relate le retour d'un écrivain en 
Haïti après vingt ans d'exil et parle de la mort, du Pays sans chapeau où vont les morts. Peu à peu, l'écrivain reprendra contact avec son Pays sans chapeau, avec ses croyances et ses mythes. Le pays réel est celui des mendiants et de l'extrême pauvreté. Le pays rêvé sera celui de l'au-delà et du vaudou, celui de ses mythes, de ses légendes : c'est la culture dont on aurait voulu le déposséder en le contraignant à l'exil et qu'il revendique lors de ce voyage. Dans ce roman où alternent les pages consacrées au " pays réel » et au " pays rêvé », le narrateur-écrivain voyage entre l'identité réelle et l'identité rêvée tant que cette dernière finit par gagner son terrain.

Il nous faut accepter qu'il y a toujours des écarts entre les intentions de l'auteur impossibles à vérifier qu'il soit en vie où dans le panthéon -, et ses réalisations. La réalité n'est jamais donnée dans l'œuvre, elle est à retracer pas à pas au cours de l'écriture et de la lecture. Il nous semble, dans ces conditions, plus rentable d'examiner la logique de ces écarts entre l'écriture et la lecture. Nous allons voir, que c'est d'autant plus valable en parlant de Dany Laferrière qu'il se qualifie lui-même écrivain-lecteur ce qui lui procure la possibilité de jouer en même temps le rôle d'un Moi qui examine et critique et le rôle de l'Autre examiné et critiqué.

7 Notre écrivain est arrivé, d'après ces propres aveux, à boucler son cycle romanesque autobiographique, ou avec une expression plus pertinente autofictionnel :

J'avais bien averti les critiques d'attendre que je termine le cycle (Une autobiographie américaine) avant de me coller toutes sortes d'étiquettes. Ils pouvaient donner au fur et à mesure leur avis (positif ou négatif) sur chaque livre paru mais je tenais encore la clé de l'ensemble. Je savais que tant que les dix livres n'étaient pas publiés, personne ne pouvait connaître le fin mot de l'histoire. (Saint-Éloi, 2001)

Qui est-ce alors cet écrivain-fantôme dont l'identité nous occupe ? Laferrière, après ses études au collège, intéressé par la peinture haïtienne et le spiralisme ${ }^{1}$, fait ses premiers pas dans le journalisme. Après la mort de son ami journaliste Gasner Raymond, tué par les tontons macoutes ${ }^{2}$, ayant appris que le même sort lui est réservé par la dictature, il se décide, en été 1976, à quitter immédiatement son pays natal. Il partagera ainsi le sort de son père auquel il se voit lié fatalement par l'histoire collective : "A dix-neuf ans, je devenais journaliste en pleine dictature des Duvalier. Mon père, lui aussi journaliste, s'était fait expulser du pays par François Duvalier. Son fils Jean-Claude me poussera à l'exil. Père et fils, présidents. Père et fils, exilés. Même destin » (Laferrière, 1999 : 132).

Il arrivera à Montréal, dans

ce nouveau monde, dit-il. Comme ça, du jour au lendemain. Un univers avec ses codes, ses symboles. Une ville nouvelle à connaître par cœur. Sans guide. Ni dieu. Les dieux ne m'accompagneront pas. L'ancien monde ne pourra m'être d'aucun secours (...). Et Montréal ne m'attend pas. (Laferrière, 2000 : 317-318)

Montréal ne signifie pas pour lui tout simplement de se plonger dans l'écriture pour se procurer un nouveau moi qui se forme tout en écrivant des récits, mais signifie également de se plonger dans des lectures lors desquelles il peut s'identifier non pas avec les personnages mais avec les écrivains eux-mêmes :

J'ai toujours rêvé d'écrire un livre plus grand que moi. Le livre que j'aimerais tant lire. (...) Je suis d'abord un lecteur. (...) Mes héros ne sont pas forcément des personnages de roman. Ce sont plutôt des écrivains. Je dirais que je préfère Borges à son œuvre, mais ce sont les livres de Borges qui ont créé le personnage. L'écrivain Borges a inventé Borges. (...) C'est l'énergie qu'ils mettent à devenir eux-mêmes qui m'impressionne. Les livres ne sont que des accessoires dans ces destins. - Se souvient-il dans une interview. (Saint-Éloi, 2001) 


$$
\text { douce Laferrière retracera, sur le mode fictionnel, son initiation à la réalité sociale et }
$$
culturelle de Montréal au tournant des années 1970-1980.

11 En 1985, le succès bruyant de son premier roman, Comment faire l'amour avec un nègre sans se fatiguer, introduit Dany Laferrière au centre de la vie culturelle québécoise.

Les auteurs d'origine haïtienne occupent actuellement une des plus importantes positions dans le domaine des écritures migrantes au Québec. Il faut noter que Daniel Chartier dans son Dictionnaire des écrivains émigrés au Québec 1800-1999 (Chartier, 2003 : 326) répertorie quarante-cinq noms d'auteurs venus d'Haïti où il dit que Dany Laferrière apparaît aujourd'hui, comme l'écrivain le plus médiatisé, dont l'œuvre est traduite et publiée dans plusieurs pays du monde.

13 La littérature haïtienne de langue française est née en un temps où l'exil des lettrés vers les centres européens était de coutume. En 1925, l'émigration vers la capitale française était une tradition bien ancrée dans la formation des intellectuels. Mais l'on a affaire ici à des vagues d'émigration temporaires, ponctuelles : l'arrivée au pouvoir de François Duvalier, en 1956, va changer la situation. Le régime dictatorial instauré en Haïti par « Papa Doc » met l'intellectuel face à une alternative décisive. Ce dernier doit choisir entre la désertion et la collaboration avec le pouvoir en place.

Lorsque « Baby Doc » (Jean-Claude, fils de François Duvalier) quitte le pouvoir, en 1986, il apparaît que le phénomène est devenu structurel et tout retour en arrière est devenu impossible.

15 À la question du journaliste : Exil ? Dany Laferrière répond les suivants : « Je ne connais pas ce mot. Cela fait vingt-cinq ans cette année que je suis en voyage. Je suis un voyageur, pas un exilé. L'exilé regarde tout le temps derrière lui ». (Saint-Éloi, 2001)

16 Laferrière fait partie donc de ces artistes émigrés qui, conçoivent l'exil comme libérateur, c'est-à-dire comme une initiation qui incite l'acte créateur et qui aide à regagner une intégrité identitaire au départ perturbée. L'écrivain affirmera en 1994 : « D'être exilé permet d'écrire sans concession et sans peur. L'exil m'a aidé à dire ce que je pense, et m'a donné la possibilité de parler à un autre pays » (Bordeleau, $1994: 10$ ). Le premier moyen pour l'écrivain de s'accrocher aux regards accueillants des habitants du pays adoptif est d'écrire pour eux.

17 C'est en ces termes suivants que le narrateur de Chronique de la dérive douce, sixième livre de Laferrière, fait le bilan de sa première année vécue en exil : «Quitter son pays pour aller vivre dans un autre pays dans cette condition d'infériorité, c'est-à-dire sans filet et sans pouvoir retourner au pays natal, me paraît la dernière grande aventure humaine » (Laferrière, 1994 : 132-133).

18 En exil, l'exilé est entre deux mondes, deux cultures et deux identités. Il a besoin de s'intégrer, ici ou là, pour pouvoir s'élaborer une nouvelle identité. Il aura du mal à effectuer ce travail d'intégration sans l'accueil et sans l'hospitalité de la culture adoptive. Il lui faut user de la langue de l'Autre. Ses textes sont marqués d'un éloignement avec la terre natale et portent en même temps celui d'un immense espoir de retour au pays natal.

19 Pour l'exilé la seule chance de pouvoir s'intégrer (non s'assimiler) dans l'ailleurs est le regard de l'autre et l'hospitalité de l'autre. Une fois intégré, l'exilé est capable d'assumer une culture plurivoque qui parle en une autre langue. Il n'est plus entre deux 
espaces culturels et identitaires mais dans les deux, avec un décalage de temps. Dany Laferrière s'intéresse à cet état de pluriculturalisme et à ces identités multiples offertes par l'accueil de l'autre et par l'écriture. Le problème, c'est dans le temps, on ne peut pas être dans deux ou plusieurs espaces à la fois, on ne peut pas avoir deux ou plusieurs identités en même temps, sauf si, grâce à la création littéraire, il ne réussit pas à arrêter le temps et à vivre chaque moment au présent. La personne Dany Laferrière aura ainsi autant d'identités que de moments vécus. L'unité intégrale de son moi se dessine en fantôme entre les moments fugitifs et entre les textes envolés.

Si la «première génération» des auteurs haïtiens tourne assez naturellement vers l'Europe - et tout particulièrement vers Paris -, la génération suivante a choisi pour cadre de sa "reprise identitaire" le continent nord-américain, grâce à la relative facilité avec laquelle on peut émigrer en Amérique du Nord, et notamment au Canada. L'espace français est devenu, au contraire, quasi impénétrable pour un immigrant en quête d'une terre d'accueil.

21 Il se forme donc une identité de génération derrière laquelle se trace le choix de la terre d'exil et ce choix assumé devient un trait d'identité. Cette identité de génération et le procédé de la prise de conscience de cette identité est définie par Pierre Bourdieu par le terme de l'opposition. Bourdieu a bien mis en évidence, en termes de fonctionnement des champs, la nécessité, pour un écrivain désireux d'entrer dans le champ littéraire, de s'y créer un espace propre et, pour ce faire, de "se poser en s'opposant» à ceux qui l'ont précédé. Notre écrivain adopte exactement cette démarche puisque la dénonciation des positions préétablies étant seule à même d'autoriser la création de positionnements neufs.

Le cas de Dany Laferrière est, à cet égard, exemplaire. Édité à Montréal, et résidant tour à tour dans la métropole québécoise et à Miami, l'écrivain revendique son américanité et l'énonce sur un axe véritablement paradigmatique : «Les gens ne viennent pas ici parce qu'ils aiment le Québec et veulent défendre le français qui est menacé en Amérique. Non, ils fuient la misère, et la politique parfois : ils viennent au Québec parce qu'on leur a dit que ça se passait mieux. Et on a beaucoup à leur offrir » (Marcotte, 1990 : 80-81).

23 Cette "américanité fondamentale " affichée par Laferrière prendra, entre autres, la forme d'un jeu référentiel et intertextuel qui place Chester Himes et James Baldwin figures incontournables de la littérature noire américaine - au cœur du dispositif textuel : avec la publication de Comment faire l'amour avec un nègre sans se fatiguer.

24 Au moment où Dany Laferrière arrive au Québec, un débat s'entame sur la société pluriculturelle. Après l'échec du premier référendum sur la souveraineté-association en 1980, l'idéologie nationaliste, dominante dans le discours social, est mise en doute. Parallèlement, on observe un apport de plus en plus dynamique de la part des écrivains venus de l'étranger. En 1983 paraît le roman La Québécoite de Régine Robin qui inaugure la discussion sur les écritures migrantes, tandis que les écrivains et intellectuels groupés autour de la revue Vice Versa lancent le concept de «transculture ».

Comment faire l'amour avec un nègre sans se fatiguer marque la dénonciation des stéréotypes raciaux. La déconstruction des codes culturels et l'intertextualité protéiforme expriment une tentative d'auto-engendrement identitaire du sujet migrant par l'intermédiaire d'une écriture libre et libérant de tout déterminisme ethnique ou idéologique. D’après Jozef Kwaterkó : 
L'opération consiste ici, à ouvrir le texte à un syncrétisme déconcertant, qui connote un univers transculturel, réfractaire à toute récupération identitaire: Henry Miller, James Baldwin, Chester Himes, Yukio Mishima, Charles Bukowski, Sylvia Plath, Jacques Roumain, Victor-Lévy Beaulieu, Sigmund Freud, Blaise Cendrars, Marguerite Duras, Marguerite Yourcenar, les musiciens noirs du jazz new-yorkais, le Kamasoutra et le Coran. (Kwaterko 2002, cité par Sadkowski, 2007 : 52-53)

L'écriture aura la fonction de faire reconnaître son identité, surtout au sens social, dans un nouvel univers que l'écrivain cherche ainsi à conquérir.

Pour le héros-narrateur la littérature sert de moyen d'exploration du monde de l'autre et de réappropriation de l'identité. À l'intérieur des multiples références intertextuelles, déjà dans le premier roman, on retrouve relativement peu d'exemples relevant de la littérature québécoise.

Il est étonnant également que l'univers blanc représenté dans le roman est presque exclusivement anglophone comme si l'auteur reprenait une des thématiques fondamentales de la littérature francophone du Canada qui consiste dans l'opposition dominant (anglais) / dominé (français). Il faut penser ici à la célèbre formule introduite dans le titre de l'essai de Pierre Vallières qui désigne les Canadiens francophones comme " Nègres blancs de l'Amérique » (Vallières, 1969).

À partir de son cycle romanesque - un livre en dix romans, vingt années d'écriture et une autobiographie américaine -, une identité spatiale apparente de Dany Laferrière se révèle curieusement après le retour au pays natal. Deux identités même à première vue, l'une américaine, l'autre haïtienne. L'œuvre littéraire, que l'auteur appelle « Une autobiographie américaine » (malgré son caractère hautement fictionnel) se répartira en deux cycles en fonction de l'espace mis au premier plan de l'action, d'un côté les villes nord-américaines et de l'autre Haïti.

La production romanesque de Dany Laferrière se construit ainsi sur un axe Amérique du Nord / Haïti (plus précisément Montréal / Haïti). À lire l'analyse d'Yves Chemla :

L'Autobiographie américaine est peut-être aussi une œuvre hyperréaliste et païenne, si l'on reprend les termes proposés jadis par Jean-François Lyotard. Le monopole narratif y est détruit, le narrateur perd ses privilèges en faisant valoir ce qu'il y a de puissance, non moindre, dans l'écoute, côté narrataire, et aussi dans l'exécution, côté narré (Chemla, 2001).

Le personnage principal, en même temps le narrateur de cette autobiographie fictive, Vieux Os oriente la lecture vers une fonction unique, celle de chercher à repérer le principe unificateur et d'extraire du livre le sens qui s'y trouve enseveli, tout en refusant au lecteur cette possibilité. Ce sens serait nommé grâce au choix d'un ensemble de citations. En fait, on aurait là des points, comme le racisme, le sexe, la mélancolie, la solitude, la religion, l'immobilité, le jazz, les influences littéraires. Le titre renvoie à l'Amérique, certes. Mais Laferrière dit aussi ceci dans l'interview déjà citée :

Je suis aussi tout ce que je ne veux pas être. Je suis un écrivain haïtien, un écrivain caraïbéen (ce qui est légèrement différent d'un écrivain antillais, mais je suis aussi un écrivain antillais), un écrivain québécois, un écrivain canadien et un écrivain afro-canadien, un écrivain américain et un écrivain afro-américain, et, depuis peu, un écrivain français. (...) Je change peut-être de chapeau mais jamais de discours (Saint-Éloi, 2001). 
C'est le discours donc qui fonde l'unité de cette quête identitaire d'écrivain et non pas le lieu où il mène ses recherches. Dans les discours de Dany Laferrière, les phrases qui commence par «Je suis... » exprime une appartenance plus universelle au monde, en dehors de celle du métier : "Je veux être pris pour un écrivain, et les seuls adjectifs acceptables dans ce cas-là sont: un bon écrivain (ce qualificatif a bien sûr ma préférence) ou un mauvais écrivain. » (Saint-Éloi, 2001).

L'écrivain, tout en s'élaborant, pendant de longues années à Montréal, une identité professionnelle finit par retrouver son intégrité syncrétique et universelle: «Il y a aussi la fatigue de quelqu'un qui n'en peut plus de toutes ces idéologies (créolité, antillanité, métissage, francophonie) qui forment comme un nuage autour de l'écrivain et l'empêchent d'apparaître devant le lecteur dans sa plus simple expression d'être pour dire tout naturellement, sans hausser le ton : je suis un homme » (ibidem).

Pour y arriver, après l'aventure américaine, l'écrivain a besoin de retourner, au sens physique et moral, dans son pays pour laisser derrière lui les identités fragmentaires et analytiques que le monde occidental, loin de Haïti, peux lui offrir.

Finalement, son identité profonde vient du temps de l'enfance et non pas des espaces parcourus, comme il dit dans l'interview déjà évoquée : «C'est un temps que je porte constamment en moi. Le pays de l'enfance » (ibidem).

Dans les romans écrits en Amérique, l'écrivain oppose Noir et Blanc, tandis que dans les récits sur Haïti, il assume une identité très personnelle haïtienne qui s'alimente de son enfance, de ces souvenirs. Apparemment, c'est toujours le regard de l'autre qui lui détermine sa position. Face à l'Européen (qu'il confond étrangement avec le Français) il assume profondément son américanité et verbalise une critique tranchante de l'hégémonie des Français: A la question du journaliste, lors du fameux entretien: «L'américanité : être écrivain américain pour toi, cela voudrait dire quoi ?» Dany Laferrière répond les suivants :

C'est habiter l'espace où je vis. C'est l'occuper émotionnellement et physiquement. Je suis un Américain, c'est-à-dire que je suis né dans le Nouveau Monde. Je connais mon territoire, comme l'animal sauvage connaît sa tanière. Je pratique un art de vivre direct, simple, franc. (...) L'Europe croit pouvoir décider de ce qui est beau. Une telle prétention me fait bondir dans l'arène. Affirmer cela c'est n'avoir aucune idée de ce qu'est la culture. Allez en Europe, vous verrez, les gens croient vraiment qu'on peut mettre la culture dans une boîte de conserve et l'envoyer aux pauvres américains (ibidem).

Le roman où Dany Laferrière s'esquisse une identité valable dans toute condition géographique, physique et morale est Pays sans chapeau.

Ce roman raconte le retour d'un écrivain en Haïti après vingt ans d'exil. Il retourne aux femmes de sa famille, sa mère et sa tante, attachées à leur terre, même aux heures les plus atroces de la dictature. L'écrivain trace par petites touches son portrait d'Haïti d'aujourd'hui et parle de la mort, du Pays sans chapeau où vont les morts.

Ce livre de Dany Laferrière est l'histoire d'un retour. Après vingt années passées à Montréal et Miami, l'auteur rentre chez lui, à Port-au-Prince, Haïti. Le pays, en apparence, est le même. L'odeur du café est la même, la pauvreté aussi, crue et violente, jusqu'aux amis qui sont restés fidèles à leur jeunesse. Mais au fil des jours, des silences de ses proches, des mots chuchotés par la rue, c'est à une enquête sur les morts que se livre l'auteur, zombis haïtiens et fantômes installés dans la vie quotidienne : 
Combien d'enfants avez-vous, madame? - Seize - Où sont-ils ? - Tous les neuf sont à l'école. - Et les autres? - Quels autres? - Les autres sept enfants. - Mais, monsieur, ils sont morts. - Madame, on ne compte pas les morts. - Et pourquoi ? Ce sont mes enfants. Pour moi, ils sont vivants à jamais (Laferrière, 1999 : 11).

À côté de ce Pays réel, il existe un Pays rêvé, le Haïti des zombis et des esprits que Vieux Os redécouvre après toutes ces années. Tout comme ces mots créoles restés en désuétude trop longtemps, qu'il faut de nouveau goûter. Ailleurs, Dany Laferrière dira : «En écrivant en français, je tue ma langue, le créole. Et personne ne m'a jamais dit: Mes condoléances. »

41 À la fois grave et léger, Pays sans chapeau se lit comme un hommage à l'imaginaire magique du peuple de Port-au-Prince. Ce roman du retour aux sources, Dany Laferrière le dédie à sa mère Marie, celle "qui n'a jamais quitté son pays, même pour une minute ». Après avoir passé 20 ans à l'étranger, à Montréal d'abord puis à Miami, l'écrivain revient en Haïti pour y redécouvrir tout ce qu'il n'avait pas oublié, qui lui manquait et qu'il n'arrive plus très bien à comprendre.

En 20 ans, il y a eu bien des massacres et des changements politiques dans l'île, mais ce n'est pas par ce biais que l'auteur aborde la réalité. C'est par le quotidien du petit peuple dans les rues, par les odeurs de cuisine à la maison, les rencontres avec ses anciens amis, avec ses anciennes flammes. Rien n'a changé : ces gens souffrent de la faim et leur esprit, fertile en superstitions, n'a jamais cessé de s'inventer des fantômes :

Dans ce pays, les morts sont partout présents, la mort semble même s'être emparée des vivants. Les atrocités de la guerre et le poids de la tyrannie dictatoriale ont donné lieu à des histoires fantastiques, à des mythes. Comme l'histoire sur les habitants d'un village du nord-ouest d'Haïti, Bombardopolis, qui n'ont pas besoin de manger pour vivre. D'après une équipe de chercheurs - venue de l'Occident pour étudier le phénomène ! - ceci doit sa raison au fait que les habitants parlent créole (Laferrière, 1999 : 194).

43 Ceux que là-bas on appelle zombis hantent les rues. La seule chose qui les différencie des vivants est leur absence de reflet dans le miroir. La mère de l'auteur lui parle longuement de ces fantômes. Des histoires entendues dans la rue viennent justifier ses peurs. L'écrivain décide donc de partir à la recherche de la vérité, rencontre d'éminents professeurs qui confirment l'existence de ces êtres. Puis quelqu'un lui propose de faire le voyage vers l'autre monde, le pays sans chapeau, car on n'a jamais enterré quelqu'un avec son chapeau... Les premières lignes du texte sont, a cet égard, limpides :

Il y a longtemps que j'attends ce moment : pouvoir me mettre à ma table de travail (une petite table bancale, sous un manguier, au fond de la cour) pour parler d'Haïti tranquillement, longuement. Et ce qui est encore mieux : parler d'Haïti en Haïti. Je n'écris pas, je parle. On écrit avec son esprit. On parle avec son corps. Je ressens ce pays physiquement. Jusqu'au talon. Je reconnais, ici, chaque son, chaque cri, chaque rire, chaque silence (idem : 13).

Tout le texte sera ainsi placé sous le signe de la culture orale. Des extraits de chansons folkloriques sont mis en épigraphe, et chaque chapitre se voit précédé d'une transcription proverbiale, dont une note préliminaire au roman précise qu'elles seront «transcrit(e)s en créole plutôt étymologique que phonétique et traduit(e)s littéralement » car, de l'avis de l'auteur, « de cette manière, leur sens restera toujours un peu secret», ce qui "nous permettra d'apprécier non seulement la sagesse populaire, mais aussi la fertile créativité langagière haïtienne » (idem : 8). 
Le narrateur prend en charge la réalité haïtienne. Vieux Os, qui a passé autant d'années en Haïti qu'il a vécu loin d'elle, est-il l'homme de deux mondes, ou un personnage horsmonde, apatride, déraciné ? Il lui faut retrouver le présent d'autrefois pour être accueilli par les siens, pour que sa propre culture d'origine le pénètre de nouveau.

Le roman se construit sur le principe d'une alternance entre des chapitres qui seront tour à tour intitulés : «Pays rêvé » et «Pays réel ». Le pays réel, c'est celui que le narrateur vit de manière quotidienne, et qu'il dessine sous forme d'une série de tableaux juxtaposés, de séquences narratives caractérisées par leur brièveté, leur hétérogénéité, et leur rapidité. Tous ces tableaux sont autant de bribes de discours s'essayant à retracer la dimension kaléidoscopique des visions et des images qui s'imposent au narrateur. C'est là une stratégie discursive familière à Dany Laferrière. Ses écrits souvent ont le caractère pictural, photographique. Si l'on accepte que l'identité psychologique se compose en premier lieu des souvenirs du passé et de l'enfance, les photos garantiraient la possibilité d'en saisir les moments. La photographie, art privilégié d'enregistrer le moment, reste tout de même une image où l'identité du photographe et du photographié glisse en fantôme parmi les prises de vue du présent: «Le temps présent - explique l'auteur dans une émission radiophonique déjà mentionnée - est encore plus poignant que le passé. Il est là et on n'arrive pas à le tenir. On tient le passé. On en fait une petite musique : la nostalgie. On a l'œil sur le futur. On y met nos espoirs, nos rêves fous avec tout de même cet air de ne pas trop y croire. » (Saint-Éloi, 2001).

Ces tableaux photographiques, instantanés servent, exactement comme les photos de famille, à élaborer des identités dans le temps chronologique et d'esquisser un passé identitaire et de le perpétuer.

Un autre moyen de se débarrasser des contraintes tyranniques du temps et de s'approcher de sa plus profonde identité psychologique, c'est la technique bien connue de la démarche proustienne. Les odeurs occupent ainsi dans la narration une place de choix, remplissant une fonction identique à celle qu'assurait ailleurs la madeleine proustienne. Ce peut être par exemple le café : «D'abord l'odeur. L'odeur du café des Palmes. Le meilleur café du monde, selon ma grand'mère. Da a passé toute sa vie à boire ce café. J'approche la tasse fumante de mon nez. Toute mon enfance me monte à la tête » (Laferrière, 1999 : 22).

Quand le narrateur part à la recherche du "pays rêvé », il ne s'agit pas ici d'un pays sublimé, idéalisé, comme une interprétation littérale pourrait le laisser penser. La dualité se concentre plutôt sur une opposition entre deux mondes, le diurne et le nocturne. Dans la nuit, le rêve se transforme en délire, en fantasme, en mythe, en légende.

Le voyage du narrateur en territoire interdit est l'occasion pour l'auteur d'affirmer qu'Haïti est devenu un " pays sans chapeau ", c'est-à-dire une terre de la mort où peine une population plus morte que vive. Ainsi que nous l'explique l'auteur en tête d'ouvrage :

Cette fine poussière sur la peau des gens qui circulent entre midi et deux heures de l'après-midi. (...) Une sorte de poudre de talc. C'est ainsi que Da me décrivait les gens qui vivaient dans l'au-delà, au pays sans chapeau, exactement comme ceux que je croise en ce moment. (...) L'au-delà. Est-ce ici ou là-bas ? Ici n'est-il pas déjà làbas? C'est cette enquête que je mène (idem : 63). 
51 Le pays réel et le pays rêvé ne s'oppose donc pas dans un ordre moral ou psychologique, mais vont signifier les deux côtés de la même réalité, d'une part la vie et d'autre part la mort. En Haïti les deux se confondent, autrement dit la mort fait partie de la vie réelle où les morts errent parmi les vivants. Le retour réel au pays réel se transforme en retour mythologique au pays des morts où la fonction de l'écriture et de la langue poétique est sacrée; elle révèle l'absolu par le truchement du mythe, qui apparaît comme seul capable de saisir globalement la réalité multiple de l'histoire et d'exprimer, à travers les fluctuations du temps humain, l'éternité.

52 L'écriture et le langage de Dany Laferrière reflète également la vision picturale primitive de la peinture haïtienne, chère à l'auteur depuis son enfance, ainsi que l'espace circulaire, le mouvement et le rythme des tambours. Comme il explique au journaliste déjà rencontré plus haut, tout cela lui n'est pas folklorique :

Ce n'est pas folklorique dans mon cas. Ce sont des trucs (le rara, la peinture naïve) que je trouve absolument intéressants, et en accord total avec ma sensibilité. Et dès qu'on trouve cet accord, on n'est plus un colonisé culturel. Il n'est pas obligatoire que cela vienne de ton pays. C'est tout simplement la rencontre explosive (genre coup de foudre) d'une forme avec une émotion. Peut-être que cela remonte à l'enfance (Saint-Éloi, 2001).

53 Si l'on accepte que notre écrivain, depuis Montréal est à la quête d'une identité, on peut conclure qu'il est possible de parler d'une effervescence du désordre identitaire qui se dessine entre les œuvres. Pour découvrir l'ordre dans le désordre apparent, le lecteur doit appliquer une lecture pure et nette des livres, et suivre pas à pas l'écrivain sur son chemin. Sauf que la direction de cette découverte mène vers l'intérieur, comme explique l'auteur lui-même en parlant de la peinture naïve :

Dans la plupart des toiles occidentales, le point de fuite est au fond du tableau. Comme une invitation à pénétrer dans le tableau. On s'installe ainsi dans l'univers du peintre, et on étudie, on flâne. Mais comme tout est sur le même plan dans la plupart des tableaux naïfs, on finit par se demander où est le pont de fuite. Je l'ai cherché jusqu'à ce que j'ai découvert que c'était mon plexus qui servait de point de fuite. Donc, voilà pourquoi je n'arrivais pas à pénétrer dans le tableau. C'est lui qui devait pénétrer en moi (Chemla, 2001).

Il faudrait peut-être, en déplaçant sensiblement la perspective inversée décrite ici, que le lecteur lui-même se reconnaisse comme « un Nègre ».

La grande contemporanéité, d'une manière paradoxale, pourrait être le résultat des retrouvailles avec le syncrétisme qui pénètre la culture et la littérature américaine de la même façon que les arts primitifs ont pénétré les arts avant-garde. L'écrivain Dany Laferrière associe en une seule entité "le peintre et l'écrivain ", et se présente comme : "Un écrivain primitif: Je n'écris pas je parle. On écrit avec son esprit (...) Une mangue tombe, j'écris mangue. Les enfants jouent au ballon dans la rue parmi les voitures. J'écris : enfants, ballon, voitures. On dirait un peintre primitif. Voilà c'est ça. (...) je suis un écrivain primitif. » (Laferrière, 1999 : 11).

Dany Laferrière, en tant que lecteur et écrivain à la fois, voltige entre les espaces géographiques et nationaux, entre les cultures américaine et haïtienne, prend la posture tantôt du moi contemplateur critique, face à l'autre, il se plait tantôt dans le rôle de l'autre critiqué et nègre : « Les gens voient souvent en moi un provocateur alors que je tente simplement de prendre des nouvelles de moi-même par le biais de l'écriture. C'est cela : j'écris pour savoir ce que je suis devenu. » (Saint-Éloi, 2001). 


\section{BIBLIOGRAPHIE}

BORDELEAU, Francine (1994). « Dany Laferrière, sans armes et dangereux », Lettres québécoises, 73. CHARTIER, Daniel (2003). Dictionnaire des écrivains émigrés au Québec 1800-1999, Québec : Éditions Nota bene.

CHEMLA, Yves (2001). Quelques notes sur Vieux Os, ce narrateur qui « porte la forme entière de l'humaine condition » inédit. Conférence donnée à UQUAM en février dans le cadre du mois des écrivains noirs.

SAINT-ÉLOI, Rodney (2001). Chronique de la retraite douce. Entretien avec Dany Laferrière, mars-août, www.lehman.cuny.edu/ile.en.ile/paroles/laferriere.html

KWATERKO, Józef (2002). «L'imaginaire diasporique chez les romanciers haïtiens du Québec ». Robert Dion (dir.). Le Québec et l'ailleurs. Études Francophones de Bayreuth, 5. Bremen : Palabres Éditions.

LAFERRIÈRE, Dany (1994). Chronique de la dérive douce. Montréal : VLB Éditeur.

LAFERRIÈRE, Dany (1999) [1996]. Pays sans chapeau. Outremont : Lanctôt éditeur, « Petite collection Lanctôt ».

LAFERRIÈRE, Dany (2000). Le Cri des oiseaux fous. Outremont : Lanctôt éditeur.

MARCOTTE, Hélene (1990). « Québec français ». Québec, 79.

SADKOWSKI, Piotr (2007). «L'écrivain ‘transaméricain' se met en scène québécoise ou Comment faire l'amour avec un nègre sans se fatiguer de Dany Laferrière ». MOREL, Pierre (dir.). Parcours québécois : Introduction à la littérature du Québec. Chişnău : Éditions Cartier.

VALLIÈRES, Pierre (1969). Nègres blancs d'Amérique. Montréal : Éditions Parti pris.

\section{NOTES}

1. Le spiralisme est un mouvement littéraire initié par Frankétienne (1936) postulant l'ancrage de l'écriture haïtienne dans la réalité spécifique du pays par la conjugaison des associations (couleurs, sons, lignes, mots) et des connexions historiques (voir: Sadkowski, 2007 : 157).

2. Tontons macoutes est le surnom générique attribué aux fonctionnaires de la milice de Duvalier dont l'objectif principal était l'élimination brutale de l'opposition politique.

\section{RÉSUMÉS}

Il existe une variété d'identités qui se proposent à l'individu tout au long de son parcours. La notion de l'identité revêt alors de diverses formes, devient dynamique, changeante et évolue à travers des conflits et des ruptures, dans le temps et dans l'espace. Dany Laferrière, écrivain québécois, est un des plus significatifs représentants de ces prises d'identités psychologique, 
nationale et professionnelle. Né à Port au Prince le 17 avril 1953, notre auteur a grandi au village de Petit-Goâve d'Haïti. Comme il est journaliste, sa vie est menacée par la dictature. Il s'exile, donc en 1976 au Canada. C'est à Montréal que l'auteur trouve une terre d'asile, qu'il devient très vite connu comme écrivain québécois et il se consacre à la littérature. L'identité naturelle de Dany Laferrière se complète alors de l'identité professionnelle au Québec, dans le pays dont la survivance culturelle est notamment confrontée au multiculturalisme. La présente étude vise à offrir l'analyse des identités dans son roman intitulé Pays sans chapeau (Editions Lanctôt, 1996) où l'auteur tente de regagner et de ressaisir son pays natal, et son identité volée par la dictature. Dans ce roman, où alternent les pages consacrées au "pays réel " et au "pays rêvé ", le narrateur-écrivain voyage entre l'identité réelle et l'identité rêvée qui finit par gagner son terrain.

There is a number of identities which present themselves to the individuals along their lives. The notion of identity takes diverse forms, becomes dynamic, changeable, and evolves through conflicts and breaks, both in time and in space. Dany Laferrière, a writer from Quebec, is one of the most important representatives of these takings of psychological, professional and national identities. Born in Port au Prince, 17 April 1953, he grew up in the village of Petit-Goâve. As a journalist, his life is menaced by the dictatorship. He thus flees to Canada in 1976. He finds asylum in Montreal and he becomes popular quickly as a Quebecois writer, as a result he turns to literature completely. The natural identity of Dany Laferrière is completed with his professional identity in Quebec, in a country whose cultural survival is confronted with multiculturalism. The present paper wishes to analyses the different identities in his novel, Pays sans chapeau (Editions Lanctôt, 1996), in which the author tries to regain and recapture his native country and his identity stolen by the dictatorship. In this novel where pages of "a real country" ; alternate with those of "a dream country", the author-narrator travels between his real identity and his dream identity regaining eventually his land.

\section{INDEX}

Mots-clés : Haïti, identités, créole, art naïf

Keywords : Haiti, identities, creole, naive art

\section{AUTEUR}

\section{ANIKÓ ÁDÁM}

Université catholique Pázmány Péter

adam.aniko[at]btk.ppke.hu 